Wilfrid Laurier University

Scholars Commons @ Laurier

Physics and Computer Science Faculty

Publications

Physics and Computer Science

1991

\title{
Resonant Steps in the Characteristics of a Josephson Junction Coupled to a Transmission Line
}

James A. Blackburn

Wilfrid Laurier University, jabjabjab@cogeco.ca

H.J.T. Smith

University of Waterloo

Niels Grønbech-Jensen

Los Alamos National Laboratory

Follow this and additional works at: https://scholars.wlu.ca/phys_faculty

\section{Recommended Citation}

Blackburn, James A.; Smith, H.J.T.; and Grønbech-Jensen, Niels, "Resonant Steps in the Characteristics of a Josephson Junction Coupled to a Transmission Line" (1991). Physics and Computer Science Faculty Publications. 43.

https://scholars.wlu.ca/phys_faculty/43

This Article is brought to you for free and open access by the Physics and Computer Science at Scholars Commons @ Laurier. It has been accepted for inclusion in Physics and Computer Science Faculty Publications by an authorized administrator of Scholars Commons @ Laurier. For more information, please contact scholarscommons@wlu.ca. 


\title{
Resonant steps in the characteristics of a Josephson junction coupled to a transmission line
}

\author{
James A. Blackburn \\ Department of Physics and Computing, Wilfrid Laurier University, Waterloo, Ontario N2L3C5, Canada \\ H. J. T. Smith \\ Department of Physics, University of Waterloo, Waterloo, Ontario N2L3C5, Canada \\ N. Grønbech-Jensen ${ }^{a}$ \\ Department of Physics and Computing, Wilfrid Laurier University, Waterloo, Ontario N2L3C5, Canada
}

(Received 22 March 1991; accepted for publication 20 May 1991)

\begin{abstract}
A novel circuit is described which functions as an electronic analog of lumped element transmission line. The circuit requires only operational amplifiers, resistors, and capacitors. This module was coupled to a Josephson junction simulator and current voltage characteristics of the combined system were recorded. Steps were observed at voltages determined by the appropriate line resonances. When the transmission line was terminated with loads less than the characteristic impedance, chaos was seen in the lower steps. Similar results were obtained by numerical integration of the corresponding system of differential equations.
\end{abstract}

\section{INTRODUCTION}

Superconducting electronics is a term generally employed to describe circuits composed of a number of Josephson junctions (or weak links) interconnected by thin film superconducting "wires". Such circuits have been designed to perform a variety of analog and/or digital functions, including analog-to-digital conversion, signal processing, and digital logic. ${ }^{1}$ The inherent high speed of Josephson junctions implies that the thin-film interconnects should be regarded as transmission lines. ${ }^{2-4}$ The elemental building block common to any superconducting electronic network is thus a Josephson junction coupled to a transmission line, which in turn is terminated by an appropriate load impedance.

Because a transmission line possesses its own resonant modes, the question naturally arises as to how such modes might manifest themselves in the characteristics of the Josephson junction. Ganz and Mercereau ${ }^{5}$ reported the appearance of steps induced in the current voltage $(I-V)$ characteristics of noncapacitive superconducting microbridges which were coupled to an open ended superconducting microstrip cavity. Smith $e t a l^{6}$ also noted constant voltage steps in the $I-V$ characteristics when an open ended stripline was connected to a capacitive junction. Longacre ${ }^{7}$ considered the case of a Josephson junction driving a series resonant tank and, again, cavity induced steps were predicted. More recently, Jensen et al. ${ }^{8}$ reported steps and chaos in numerical simulations of a Josephson junction coupled to resonators, while Larsen et al., ${ }^{9}$ observed steps in experimental data obtained from $\mathrm{Nb}-\mathrm{Al}_{2} \mathrm{O}_{3}-\mathrm{Nb}$ tunnel junctions embedded in open-ended superconducting strip lines.

The configuration which will be treated here consists of a Josephson weak link directly coupled to a loaded transmission line as illustrated in Fig. 1. The resistively shunted junction is modeled by means of an appropriate analog electronic circuit, while the transmission line is simulated with a novel network of operational amplifiers (op amps). The combined modules are biased by a fixed current and the resulting time dependent voltages, or their time averages, are measured with a computer based data acquisition system. This approach is a great deal faster than numerical simulation, allowing complete $I-V$ curves to be generated in only a few minutes.

\section{TRANSMISSION LINE SIMULATION}

A transmission line can be characterized by the parameters: series inductance $L(\mathrm{H} / \mathrm{m})$, shunt capacitance $C(\mathrm{~F} /$ $\mathrm{m})$, series resistance $R_{s}(\Omega / \mathrm{m})$, and shunt conductance $G_{p}(\Omega \mathrm{m})^{-1}$. A lumped element approximation to this continuous line, with cells of unit length, is shown in Fig. 2. Applying Kirchhoff's laws gives the following equations

$$
I_{(n)}-I_{(n+1)}=C \frac{d V_{(n)}}{d t}+\frac{V_{(n)}}{R_{p}}
$$

where $R_{p}=G_{p}^{-1}$ and

$$
V_{(n)}-V_{(n+1)}=I_{(n+1)} R_{s}+L \frac{d I_{(n+1)}}{d t} .
$$

As a preamble to a discussion of the complete simulation circuit, consider first the elementary op-amp configuration shown in Fig. 3. The inverting input is a virtual ground, and so equating currents arriving at this node and currents leaving it, yields

$$
\frac{d V_{\text {out }}}{d t}=-\frac{1}{C}\left(\frac{V_{x}}{R_{x}}+\frac{V_{y}}{R_{y}}\right)-\frac{V_{\text {out }}}{r C}
$$

a) Permanent address: Physics Lab I, Technical University of Denmark, Lyngby, Denmark. 


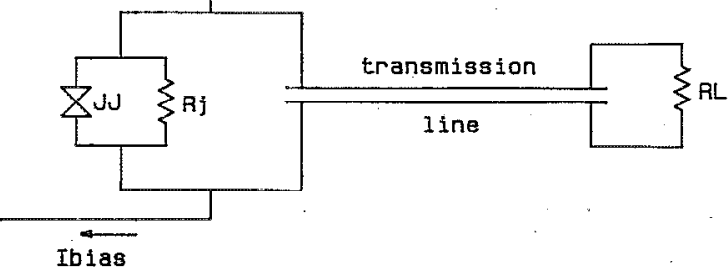

FIG. 1. Josephson junction with shunt resistor $R_{J}$ coupled to a loaded transmission line.

Figure 4 depicts the intermediate portion of a long ladder which will simulate the transmission line. It is comprised entirely of op-amp configurations of the type described above, together with simple inverters. The two left hand node voltages are labeled $V_{(n)}$ and $-V_{(n+1)}$, as shown. Furthermore, the indicated right hand node voltages are identified as $I_{(n)} R$ and $-I_{(n+1)} R$. All unlabeled nodes on both sides of the ladder are at virtual ground. Bearing in mind Fig. 3 and Eq. (3), one may write for this ladder,

$$
\frac{d V_{(n)}}{d t}=\frac{\left(I_{(n)}-I_{(n+1)}\right)}{C_{\bar{c}}}-\frac{V_{(n)}}{R_{c} C_{c}}
$$

and

$$
R \frac{d I_{(n+1)}}{d t}=\frac{\left(V_{(n)}-V_{(n+1)}\right)}{R C_{a}}-\frac{R I_{(n+1)}}{R_{a} C_{a}} .
$$

A comparison of Eq. (4) with Eq. (1), and of Eq. (5) with Eq. (2), reveals that the ladder circuit is, in effect, an
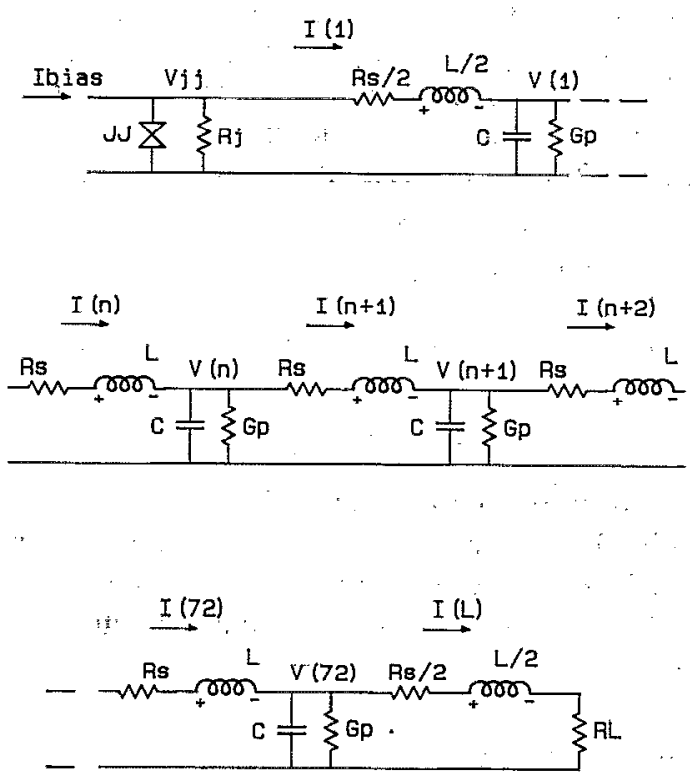

FIG. 2. Iosephson junction and lumped element transmission line composed of 72 segments.

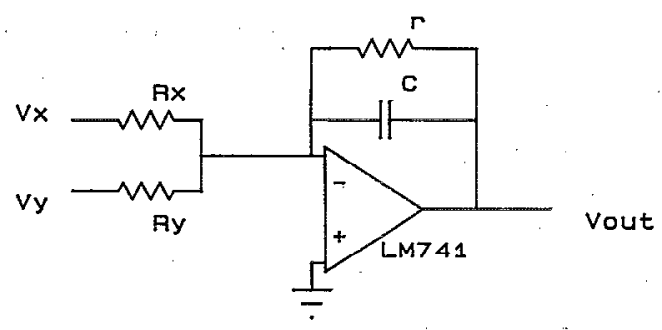

FIG. 3. General configuration of a two-input integrator with shunt resistor across the capacitor.

electronic analog computer for solving-Kirchhoff's loop and node conservation laws, with the following equivalences:

$$
C_{c}=C, \quad R_{c}=R_{p}, \quad R^{2} C_{a}=L, \quad R^{2} / R_{a}=R_{s} .
$$

As can be seen from these expressions, all parameters relevant to the transmission line are controlled by three resistors and two capacitors-no actual inductors are required. It is possible to simulate a transmission line of stipulated length by constructing the ladder circuit with an appropriate combination of $C_{c}, R_{c}, C_{a}, R_{a}$, and $R$, and by choosing a suitable value for the total number of sections in the ladder. All node voltages along the lumped element line are generated down the left hand side of the ladder, while voltages down the right hand side are proportional to the link currents. This approach to modeling a transmission line is quite general and could prove useful in studies of systems other than the particular one which we shall now consider.

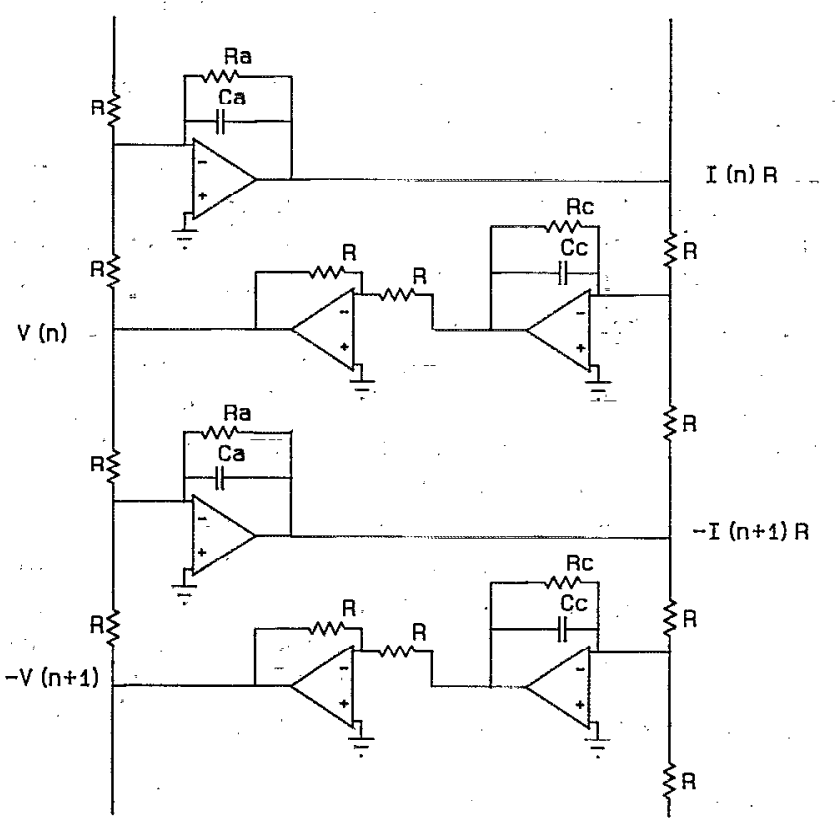

FIG. 4. Portion of the ladder circuit showing the assignment of node voltages down the left-hand side, and link currents down the right-hand side. 

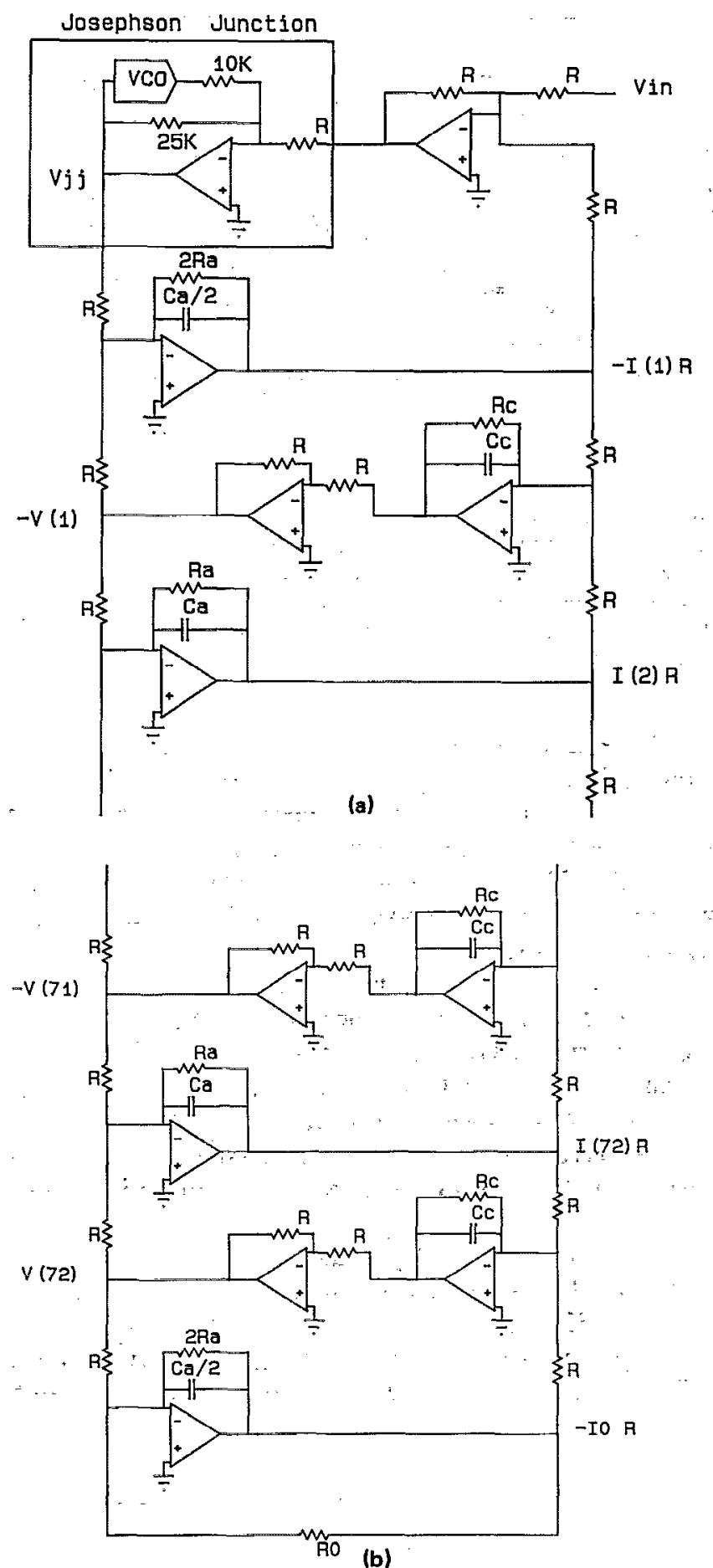

FIG. 5. (a) Input stage of the ladder circuit, (b) final stage of the ladder circuit showing the location of the termination resistor $R_{0}$.

\section{THE COMPLETE SYSTEM}

As noted earlier, we are interested in the behavior of a current biased Josephson junction which directly drives a loaded transmission line. The ladder circuit described in the previous section must therefore be connected at one end to an appropriate Josephson junction electronic simulator, and on the other end to suitable load. Figure 5 depicts the first and last segments of the circuit. The Josephson junction simulator enclosed in a box in Fig. 5(a), consists of an op-amp and voltage-controlled oscillator (VCO). Detailed descriptions of this circuit and its operation have already been published. ${ }^{10}$

The node and loop equations at the driven end of the lumped line (Fig. 2) are

$$
I_{\text {bias }}=I_{J J}+I_{(1)}
$$

and

$$
V_{J J}-V_{(1)}=I_{(1)} \frac{R_{s}}{2}+\frac{L}{2} \frac{d I_{(1)}}{d t},
$$

where $R_{s} / 2$ and $L / 2$ appear because only one branch of the lumped element pi section is present at the input to the transmission line. These values are reflected in the first integrator of Fig. 5(a) where the inductance is set by $C_{a} / 2$ and the dissipation by $2 R_{a}$. The constraint expressed by Eq. (6) is imposed at the inverting node of the input op amp, while Eq. (7) is satisfied by the first ladder op amp. The bias current is given by $I_{\text {bias }}=V_{\text {in }} / R$.

The loop equation at the termination end of the line is

$$
V_{(N)}-V_{\text {load }}=I_{(N+1)} \frac{R_{s}}{2}+\frac{L}{2} \frac{d I_{(N+1)}}{d t} .
$$

But $I_{(N+1)}=I_{\text {load }}$ and $V_{\text {load }}=I_{\text {load }} R_{L}$, where $R_{L}$ is the load resistor, and so *

$$
V_{(N)}=I_{\text {load }} R_{L}+I_{\text {load }} \frac{R_{s}}{2}+\frac{L}{2} \frac{d I_{\text {load }}}{d t}
$$

Again with reference to Fig. 3 and Eq. (3) we obtain from the final section of the simulator [Fig. 5(b)]

$$
R \frac{d I_{0}}{d t}=\frac{2 V_{(N)}}{R C_{a}} \div \frac{2 I_{0} R}{R_{0} C_{a}}-\frac{R I_{0}}{R_{a} C_{a}}
$$

which can be rearranged as

$$
V_{(N)}=I_{0} \frac{R^{2}}{R_{0}}+I_{0} \frac{R^{2}}{2 R_{a}}+\frac{R^{2} C_{a}}{2} \frac{d I_{0}}{d t} .
$$

Comparing Eqs. (11) and (9), it is apparent that the transmission line load $R_{L}$, and the simulator termination $R_{0}$, are related as follows:

$$
R^{2} / R_{0}=R_{L}
$$

Component values used in the actual circuit were: $R=39$ $\mathrm{K} \Omega, R_{a}=10 \mathrm{M} \Omega, R_{c}=10 \mathrm{M} \Omega, C_{c}=1 \mathrm{nF}, C_{a}=1 \mathrm{nF}$. Each segment has been taken to be of unit length. If, for example, the length scale is chosen to be centimeters, then the equivalent lumped element transmission line parameters would be interpreted as: $C=1 \mathrm{nF} / \mathrm{cm}, L=1.52 \mathrm{H} / \mathrm{cm}$, $R_{p}=10 \mathrm{M} \Omega \mathrm{cm}, R_{s}=152 \Omega / \mathrm{cm}$. The ladder consisted of 72 sections and so required 218 op amps, not counting the Josephson junction subcircuit. A lumped line of this length has a cutoff frequency ${ }^{11}$ of $\omega_{c}=2 / \sqrt{ }(L C)=8.164 \mathrm{KHz}$. The characteristic impedance of a corresponding continuous transmission line is ${ }^{12} Z_{0}=\sqrt{ }(L / C)=39 \mathrm{~K} \Omega$, and the phase velocity is $v_{\phi}=1 / \sqrt{ }(L C)=25650 \mathrm{~cm} \mathrm{~s}^{1}$.

If a transmission line is driven at one end, and is shorted at the other, then resonances will occur when the line contains $1 / 4,3 / 4,5 / 4, \ldots$ wavelengths. For the present 
case with an overall length of 72 , the corresponding resonant frequencies will be given by

$$
f_{n}=n \frac{v_{\phi}}{288} \quad n=1,3,5,7, \ldots,
$$

or $f_{R}=89.06,267.19,445.31 \mathrm{~Hz}, \ldots$.

If the transmission line is open ended, then resonances occur when the line contains $1 / 2,1,3 / 2,2,5 / 2, \ldots$, wavelengths, and so

$$
f_{R}=n \frac{v_{\phi}}{288} \quad n=2,4,6, \ldots,
$$

or $f_{R}=178.13,356.25,534.38 \mathrm{~Hz}, \ldots$.

Note that the first ten resonances are located at frequencies which are less than $20 \%$ of the cutoff $\omega_{c}$ quoted above. This assures that the lumped element circuit is a good approximation to the continuous line over such a frequency interval.

The VCO used in the Josephson junction simulator produced a maximum output amplitude of $0.85 \mathrm{~V}$. Since this was fed through a $10 \mathrm{~K} \Omega$ resistor, the equivalent Josephson supercurrent was $85 \mu \mathrm{A}$. The anticipated critical input bias voltage to the system as shown in Fig. 5(a) would then be $3.32 \mathrm{~V}$ (i.e., $3.32 \mathrm{~V}$ divided by $39 \mathrm{~K} \Omega$ equals $85 \mu \mathrm{A})$. The VCO sensitivity was determined to be 1080 $\mathrm{Hz} / \mathrm{V}$. As shown in Fig. 5(a), the junction shunt resistance was $25 \mathrm{~K} \Omega$.

\section{EXPERIMENTAL RESULTS}

In order to obtain a current-voltage $(I-V)$ characteristic for the system, it is necessary to measure the time averaged junction voltage $\left\langle V_{J J}\right\rangle$ while slowly incrementing the input voltage $V_{\text {in }}$ (and hence $I_{\text {bias }}$ ). The junction voltage was averaged with a simple $R C$ filter having a time constant of about $1 \mathrm{~s}$. Data acquisition was performed by a desktop computer equipped with a Scientific Solutions ${ }^{13}$ LabMaster card, and was controlled by the Labtech Notebook $^{14}$ software package. With this setup, a predefined datafile could be used to generate a bias voltage ramp from one of the D/A channels on the LabMaster board with user specified amplitude and period. Amplitudes up to 10 $V$ were used, and sweep times were typically about $10 \mathrm{~min}$. The filtered junction voltage was measured on an input A/D channel to 12 bits precision at 5 samples/s.

Two representative $I-V$ curves are shown in Fig. 6 for transmission line termination resistors $R_{L}$ smaller than the characteristic impedance of $39 \mathrm{~K} \Omega$. Both upward and downward bias sweeps were carried out, revealing considerable hysteresis in the curves. Vertical markers have been placed at voltages corresponding to $178,356,534 \mathrm{~Hz}, \ldots$. The conversion from frequency to equivalent voltage is simply done using the VCO calibration of $1080 \mathrm{~Hz} / \mathrm{v}$. Note that the bottom of each step begins at one of the marked voltages. Each step then rises with a finite slope (the commonly encountered term "constant voltage step" is obviously not really appropriate), reaching its peak at a voltage halfway to the next marker, after which a sudden transition takes place to a point on the next step. Thus, for the

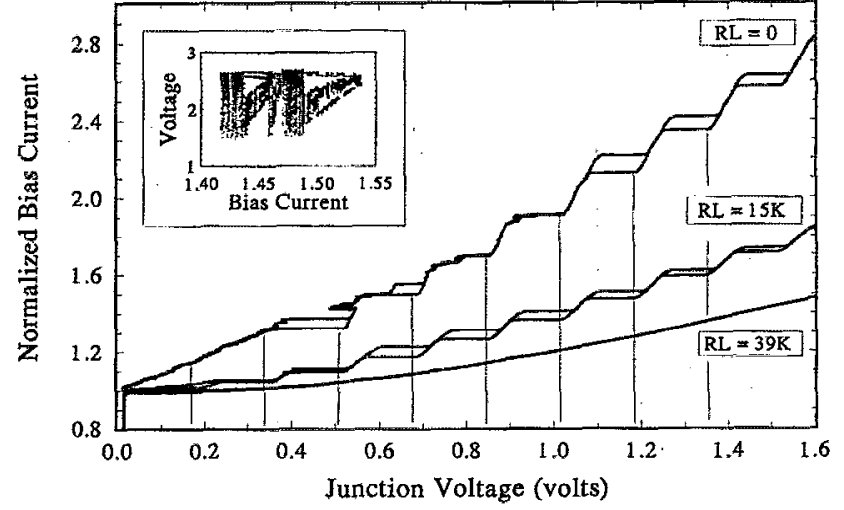

FIG. 6. Current-voltage characteristics of the combined Josephson junction and transmission line. The vertical scale is in units of the junction critical current $I_{c}=85 \mu \mathrm{A}$. The data were obtained directly from the simulator circuit for three different load resistors. (Inset) Bifurcation diagram spanning a small range of bias current for the $R_{L}=0\left(R_{0}\right.$ = open) termination.

case $R_{L}<Z_{0}$, the step maxima are located at frequencies (voltages) determined by Eq. (12) and the step width is 89 $\mathrm{Hz}$ (or $0.083 \mathrm{~V}$ ). The third characteristic in this figure was observed when the transmission line had a matched load $\left(R_{L}=Z_{0}\right)$. This termination prevents reflections at the end of the line, which now appears infinitely long, eliminating resonances and steps.

A clearer view is presented in Fig. 7 which depicts a magnified portion of one of the $I-V$ curves in Fig. 6 . It is easily seen that the distance from the top of one step to the bottom of the next is $0.083 \mathrm{~V}$. The central step begins at about $0.85 \mathrm{~V}$ and, as noted above, has a width of $0.083 \mathrm{~V}$. This observed step width may be understood by next considering the frequency dependence of the transmission line itself.

A circuit simulation package (MicroCap $\mathrm{III}^{15}$ ) was used to model a lumped transmission line of 72 segments, with $L=1.52 \mathrm{~h}, C=1 \mathrm{nF}, R_{s}=150 \Omega$, and $R_{p}=10 \mathrm{M} \Omega$. The output from MicroCap III, for $R_{L}=15 \mathrm{~K} \Omega$, is shown in Fig. 8. The frequency range has been chosen to match

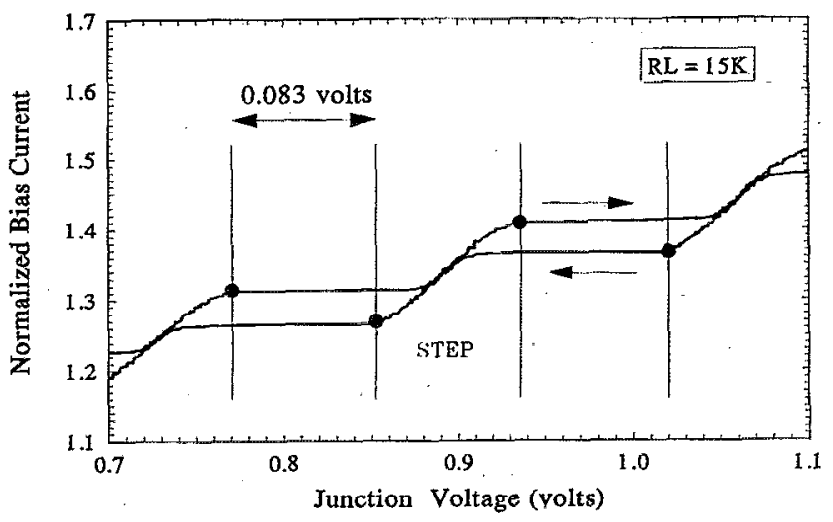

FIG. 7. Expanded portion of the $I-V$ characteristic for $R_{L}=15 \mathrm{~K} \Omega$. Note the comparatively small residual instrumentation noise. Dots mark the beginning and end of the voltage steps. 


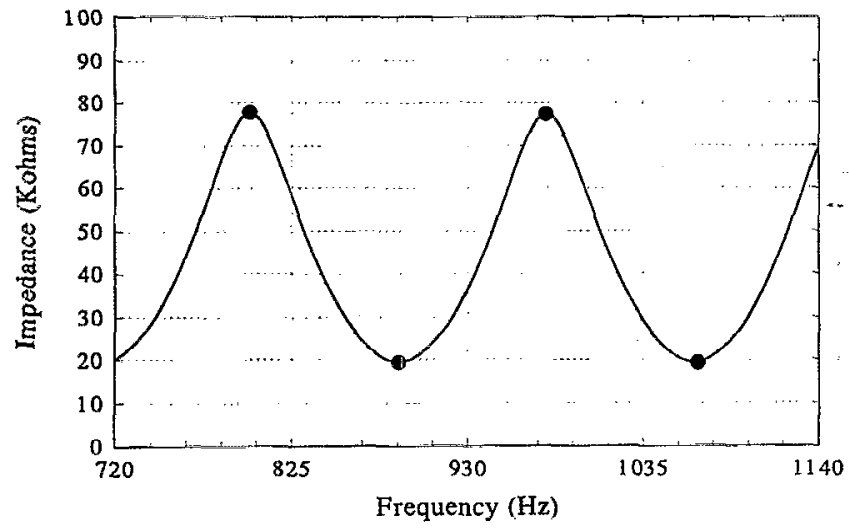

FIG. 8. Plot of the input impedance of a 72 segment lumped element transmission line with $L=1.52 \mathrm{~h}, C=1 \mathrm{nF}, R_{s}=152 \Omega, R_{p}=10 \mathrm{M} \Omega$, and $R_{L}=15 \mathrm{~K} \Omega$. These data were generated with the Micro-Cap III simulation package. The frequency range has been chosen to match the the voltage range of the preceding figure. Dots mark the impedance maxima and minima.

the voltage range of Fig. 7. A comparison of these two figures reveals that the step corresponds to the portion of the impedance curve with positive slope. The shape and width of a step is determined by the shape of the impedance curve; varying the load $R_{L}$ would alter the sharpness of the impedance maxima, and thus affect the $Q$ of the voltage steps.

Figure 9 is a companion to Fig. 6 . Here the $I-V$ characteristics are plotted for termination resistors larger than the characteristic impedance. Step maxima are now located at frequencies defined by Eq. (13), that is $178,356 \mathrm{~Hz}$, etc., corresponding to integer half wavelength modes.

Finally, we turn to the issue of noise in the $I-V$ characteristics. As can be seen in Fig. 7, the intrinsic instrumentation noise attributable to the electronics and/or the data acquisition is considerably smaller than the noise which is visible in the traces of Fig. 6. This is particularly true in the case of the $R_{L}=0$ characteristic. As an insight into the nature of this noise, a bifurcation diagram was obtained experimentally by sampling the instantaneous

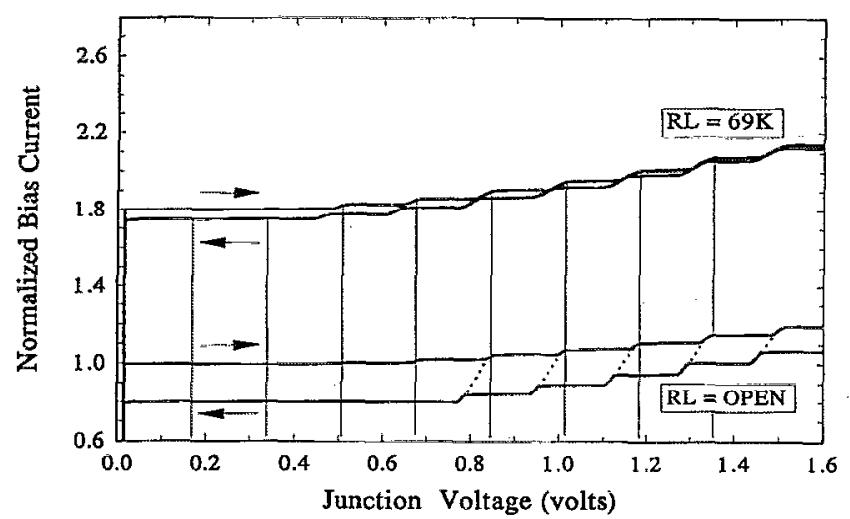

FIG. 9. Current-voltage curves from the electronic simulator showing the appearance of steps when $R_{L}>Z_{0}$. The characteristic for $R_{L}=69 \mathrm{~K} \Omega$ has been displaced upward by 0.8 units for clarity. junction voltage once in each oscillation of the junction supercurrent. This was accomplished by generating strobe pulses from the VCO and using them to trigger each A/D conversion on the Scientific Solutions board. The inset to Fig. 6 is such a bifurcation diagram containing 20000 points spanning a narrow range of bias current. There is clear evidence of multiperiodic, as well as chaotic, behavior and this suggests that for $R_{L}<Z_{0}$, low-order steps may exhibit chaos. At higher average voltages, the supercurrent wave form in a resistively shunted Josephson junction is more nearly sinusoidal. We believe that this property is connected to the absence of chaos in the higher order steps. No evidence of chaos is present in the $I-V$ curves for $R_{L}>Z_{0}$.

\section{NUMERICAL SIMULATIONS}

As an independent check of the results obtained from the simulator circuit, we carried out numerical integrations of the differential equations which govern the coupled Josephson junction and transmission line. The well known equation of motion for the phase difference across a current biased Josephson junction is

$$
\frac{d \phi}{d t}=i_{j j}-\sin (\phi)
$$

Here dimensionless currents are expressed in terms of the junction critical current $I_{c}$. Time is normalized in units of the characteristic period $\tau=\hbar /\left(2 e I_{c} R_{j}\right)$, where $R_{j}$ is the junction shunt resistance and $i_{j j}$ is the total current flowing through the Josephson junction. The dimensionless form of Eq. (6) is just $i_{b}=i_{j j}+i_{(1)}$, where $i_{(1)}$ is the input current to the transmission line.

The differential equation governing the transverse voltage across a continuous transmission line is

$$
\frac{d^{2} V}{d x^{2}}=L C \frac{d^{2} V}{d t^{2}}+\left(L G_{p}+R_{s} C\right) \frac{d V}{d t}+R_{s} G_{p} V .
$$

Transforming to the same normalized time units as were used in Eq. (14), replacing the second-order spatial derivative with the central difference expression

$$
\frac{d^{2} v}{d x^{2}}=\frac{\left(v_{n+1}-2 v_{n}+v_{n-1}\right)}{\Delta x^{2}}
$$

and rearranging

$$
\begin{aligned}
\frac{d^{2} v_{n}}{d t^{2}}= & \frac{\tau^{2}}{L C}\left[\left(v_{n+1}-2 v_{n}+v_{n-1}\right)-\left(\frac{R_{s} C+G_{p} L}{\tau}\right) \frac{d v_{n}}{d t}\right. \\
& \left.-R_{s} G_{p} v_{n}\right],
\end{aligned}
$$

where $v=V /\left(I_{c} R_{j}\right)$. To match the numerical calculation with the analog simulation, we have chosen $\Delta x=1$. Let there be $M$ nodes. Equation (16) then becomes a set of 


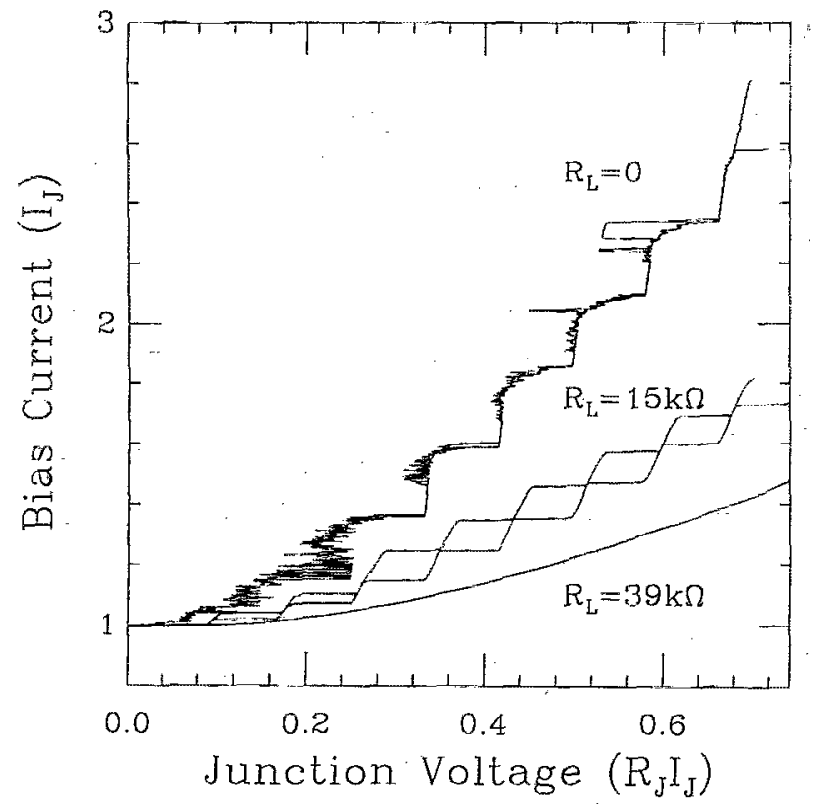

FIG. 10. Current-voltage characteristics from numerical simuTations. $\mathrm{Pa}$ rameters were chosen to correspond to the electronic simulator data shown in Fig. 6.

coupled differential equations for $n=(2, M-1)$. The first and last points are special and are governed by

$$
\begin{aligned}
\frac{d^{2} v_{1}}{d t^{2}}= & \frac{\tau^{2}}{L C}\left[\left(v_{2}-3 v_{1}\right)-\left(\frac{R_{s} C+G_{p} L}{\tau}\right) \frac{d v_{1}}{d t}-R_{s} G_{p} v_{1}\right. \\
& \left.-2 i_{1}+2\left(i_{b}-\sin \phi\right)\right] \\
\frac{d^{2} v_{M}}{d t^{2}}= & \frac{\tau^{2}}{L C}\left[\left(v_{M-1}-3 v_{M}\right)-\left(\frac{R_{s} C+G_{p} L}{\tau}\right) \frac{d v_{M}}{d t}\right. \\
& \left.-R_{s} G_{p} v_{M}+i_{L}\left(\frac{2 R_{L}}{R_{j}}\right)\right],
\end{aligned}
$$

where $i_{L}=i_{M+1}$ is the normalized current through the load resistor $R_{L}$. Finally, the currents $i_{1}$ and $i_{L}$ obey the following equations which arise from Kirchhoff's laws applied to the first and last segments

$$
\begin{aligned}
& \frac{d i_{1}}{d t}=\frac{2 \tau R_{j}}{L}\left[i_{b}-i_{1}\left(1+\frac{R_{s}}{2 R_{j}}\right)-\sin \phi-v_{1}\right] \\
& \frac{d i_{L}}{d t}=\frac{2 \tau R_{j}}{L}\left[v_{M}-i_{L}\left(\frac{R_{s}}{2 R_{j}}+\frac{R_{L}}{R_{j}}\right)\right] .
\end{aligned}
$$

This system of equations $(14,16-20)$ was solved for the set of parameters $M=72, R_{j}=25 \mathrm{~K} \Omega, I_{c}=86 \mu \mathrm{A}, L=1.52 \mathrm{H}$, $C=1 \mathrm{nF}, R_{s}=150 \Omega$, and $G_{p}=0$. Since the analog of $\hbar / 2 e$ for the electronic Josephson circuit is $(2 \pi b)^{-1}$, where $b \approx 1000 \mathrm{~Hz} / \mathrm{V}, \tau=75 \mu \mathrm{s}$. The numerical integrations were performed using a fourth-order Runge-Kutta algorithm with a normalized time step of $\Delta t=0.01$. The bias current was varied in steps of 0.003 and for each measurement of the resulting voltage, averaging over a few thousand time units was carried out.

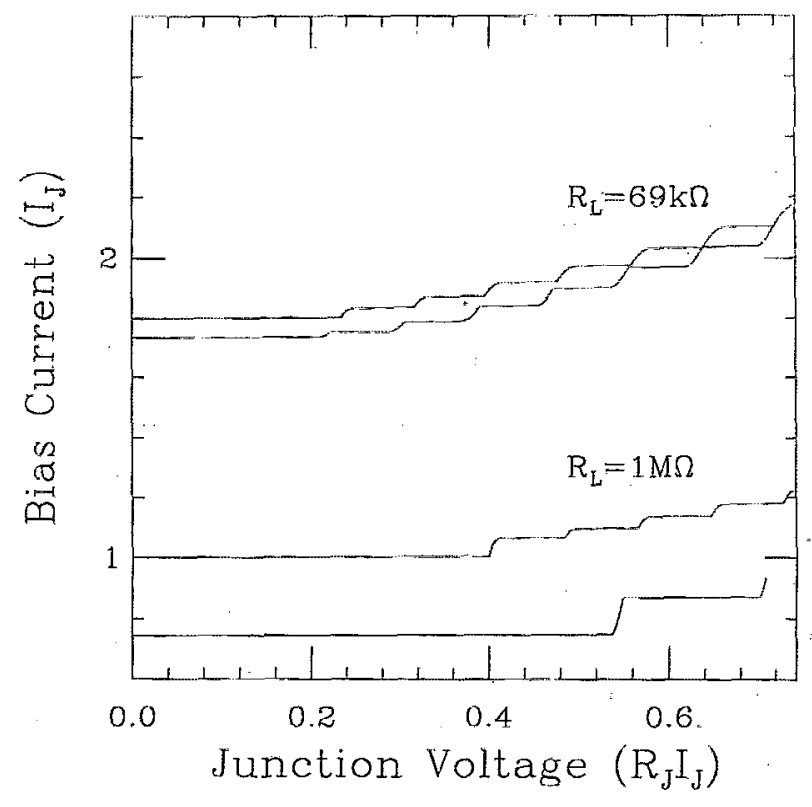

FIG. 11. Current-voltage characteristics from numerical simulations. Parameters were chosen to correspond to the electronic simulator data shown in Fig. 9.

Figures 10 and 11 show numerical results which correspond to the analog simulation data in Figs. 6 and 9. As expected, we find good agreement between the two methods. An important point to note is the significant speed difference between the two techniques. $I-V$ curves, such as those presented in Fig. 10, required several days of vector processor CPU time, yet were completed in only about 10 min on the electronic simulator! A clear artifact of the shorter averaging time in the numerical calculations is seen in the rather large variations in average $(\mathrm{dc})$ voltage when the dynamical states are chaotic.

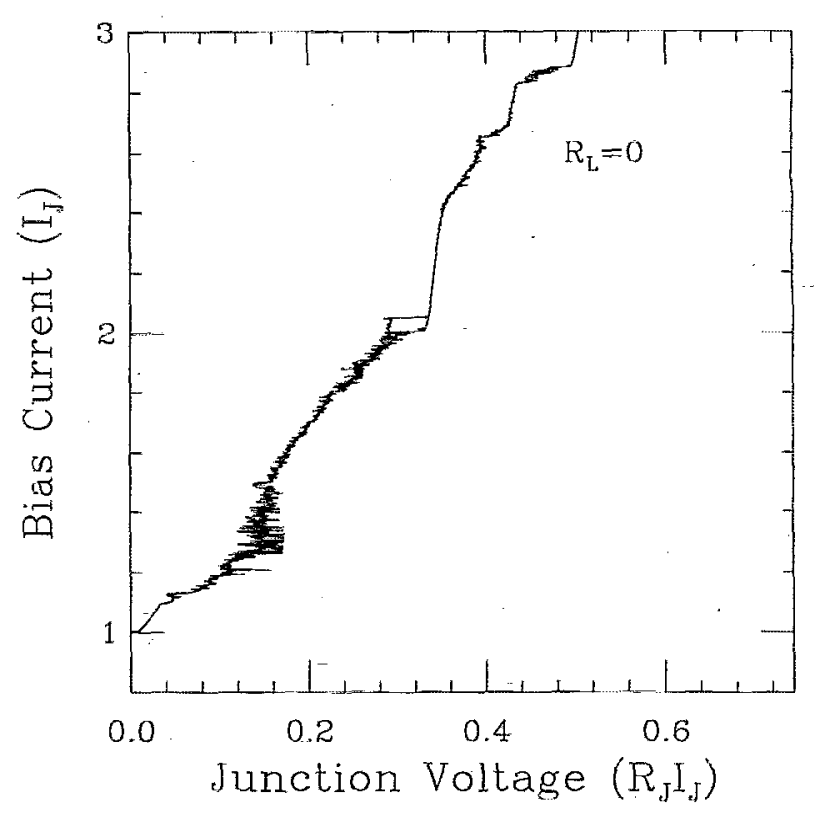

FIG. 12. Numerical simulation using a half-length transmission line. 
One slight difference between the $I-V$ curves for $R_{L}$ $=0$ is that there appears to be a finite initial slope at $i_{b}$ $>1$ in the analog simulation data, whereas the numerical simulation produces zero (or at least a very small) slope. We believe that this discrepancy is of minor importance since both characteristics display the same chaos for the low voltage steps, as well as more regular behavior at higher voltage steps. The origin of the discrepancy is most likely the fact that the numerical simulation is "ideal," whereas components in the electronic circuit are subject to offsets, thermal drift, parameter variations, and so forth. In the electronic circuit we can thus expect more complex behavior, since reflections can occur at imperfections. An elementary way to study these reflections is to consider a shorter transmission line, in which case the loaded end of the shorter line effectively models an imperfection. An $I-V$ curve was calculated using half the previous number of segments, and the characteristic shown in Fig. 12 resulted. Chaos is still present, but the initial slope for $i_{b}>1$ is now positive. For a transmission line with a distribution of imperfections from which scattering takes place, further modifications in the $I-V$ curve would be anticipated.

\section{ACKNOWLEDGMENTS}

Two of the authors (J. A. B. and H. J. T. S.) received financial support from the Natural Sciences and Engineer- ing Research Council of Canada. N. G.-J. received funding from Otto Mønsteds Fond, Christian og Ottilia Rrorsons Rejselegat.

${ }^{1}$ Superconducting Electronics, edited by $\mathrm{H}$. Weinstock and M. Niseñoff NATO ASI Series F, Vol. 59 (Springer; New York, 1989).

${ }^{2}$ H. H. Zappe, Appl. Phys. Lett. 27, 432 (1975).

${ }^{3}$ H. H. Zappe, IEEE Trans. Magn. MAG-13, 41 (1977).

${ }^{4} \mathrm{~T}$. VanDuzer and C. W. Turner, Principles of Superconductive Devices and Circuits (Elsevier, New York, 1981), pp 131-138.

${ }^{5}$ T. Ganz and J. E. Mercereau, J. Appl. Phys. 46, 4986 (1975).

${ }^{6}$ A. D. Smith, B. J. Dalrymple, A. H. Silver, R. W. Simon, and J. F. Burch, IEEE Trans. Magn. MAG-23, 796 (1987).

${ }^{7}$ A. Longacre, Jr., J. Appl. Phys. 50, 6451 (1979).

${ }^{8}$ H. Dalsgaard Jensen, A. Larsen, and J. Mygind, Physica B 165\&166, 1661 (1990).

${ }^{9}$ A. Larsen, H. Dalsgaard Jensen, and J. Mygind, Physica B 165\&166, 103 (1990).

${ }^{10}$ Wu Binruo, Yang Zhou-jing, J. A. Blackburn, S. Vik, H. J. T. Smith, and M. A. H. Nerenberg, Phys. Rev. B 37, 3349 (1988).

${ }^{11}$ J. M. Blair, Am. J. Phys. 50, 753 (1982).

${ }^{12}$ J. J. Karakash, Transmission Lines and Filter Networks (MacMillan, New York, 1950).

${ }^{13}$ Scientific Solutions, Inc., 6225 Cochran Road, Solon, $\mathrm{OH}$.

${ }^{14}$ Labtech Notebook is a product of Laboratory Technologies Corp., 400 Research Drive, Wilmington, MA.

${ }^{15}$ Micro-Cap III is a product of Spectrum Software, $1021 \mathrm{~S}$. Wolfe Road, Sunnyvale, CA. 